\title{
Psychometric Analysis of the Children's Eating Attitudes Test in Youth with Overweight/Obesity
}

\author{
Jeffrey M. Girard \\ University of Mississippi Medical Center \\ Marissa A. Gowey \\ University of Alabama at Birmingham
}

\author{
Crystal S. Lim \\ University of Mississippi Medical Center \\ David M. Janicke \\ University of Florida
}

\begin{abstract}
Objective: Disordered eating attitudes and behaviors play a critical role in pediatric overweight and obesity and are important to measure in research and practice with this population. Disordered eating attitudes and behaviors in youth are commonly measured using the Children's Eating Attitudes Test (ChEAT), which has not been psychometrically evaluated for use with youth with overweight and obesity. As such, it currently unknown whether the ChEAT provides a reliable measurement of a single underlying construct when applied in this population. Methods: Data from 400 treatment-seeking youth (aged 8 to 18) with overweight and obesity were drawn from two sites in the southern United States (54\% female, 57\% White). Psychometric properties of the ChEAT were evaluated in this combined sample using confirmatory bifactor modeling. Results: A confirmatory bifactor model with five group factors provided the best fit to the data and was used to generate numerous psychometric coefficients. The explained common variance (.36), omega hierarchical (.58), factor determinacy (.86), and construct replicability (.70) coefficients were all lower than recommended cutoff criteria. Both multidimensionality and excessive residual variance contributed to the ChEAT's poor psychometric performance in this sample. Conclusions: The ChEAT displayed marked psychometric problems in a large and diverse sample of youth with overweight and obesity. Its common variance was not unidimensional, its unit-weighted total score was not a reliable indicator of a broad central construct, and its optimally weighted total score was not likely to replicate across studies. We recommend using alternatives to the ChEAT when conducting research and practice with youth with overweight and obesity.
\end{abstract}

\section{Introduction}

Pediatric overweight and obesity $(\mathrm{OV} / \mathrm{OB})$ are increasingly prevalent public health concerns. The proportion of children and adolescents in the United States with a body mass index (BMI; $\mathrm{kg} / \mathrm{m}^{2}$ ) at or above the 85 th percentile (i.e., overweight) has increased from $29 \%$ in $1999-2000$ to $35 \%$ in $2015-2016$, and the proportion at or above the 95 th percentile (i.e., obesity) has increased from $15 \%$ to $19 \%$ (Skinner et al., 2018). Thus, one in three American youth has overweight and nearly one in five has obesity. Similar trends have been found in many countries worldwide and are most pronounced in economically developed countries and in urbanized populations (Wang and Lobstein, 2006). These numbers are concerning given that pediatric $\mathrm{OV} / \mathrm{OB}$ is associated with numerous negative health outcomes (e.g., car-

Correspondence concerning this article should be addressed to Crystal S. Lim, Department of Psychiatry and Human Behavior, University of Mississippi Medical Center, 2500 North State Street, Jackson, MS, 39216, USA. email: cstacklim@umc.edu diovascular disease and glucose insensitivity) and psychosocial sequelae (e.g., low self-esteem and academic problems) (Daniels, 2006; Sahoo et al., 2015).

Pediatric OV/OB is also associated with increased risk of disordered eating symptoms such as excessive concerns about body weight/shape, binge eating, and unhealthy weight-control strategies (Goldschmidt et al., 2008). Some degree of body dissatisfaction is common in youth across weight categories (Neumark-Sztainer et al., 2002), and a moderate degree of concern about body weight/shape may help motivate engagement in healthy weight-control strategies (Heinberg et al., 2001). However, high levels of disordered eating symptoms predict increased weight-gain over time (Neumark-Sztainer et al., 2006; Tanofsky-Kraff et al., 2006); are risk factors for full-syndrome eating disorders such as anorexia nervosa, bulimia nervosa, and binge eating disorder (Kotler et al., 2001); and can create barriers to treatment, such as when body dissatisfaction contributes to the avoidance of physical activity (Zabinski et al., 2003) or when unhealthy weight-control strategies take the place of healthy strategies (Neumark-Sztainer et al., 2004).

Given the higher prevalence of disordered eating symp- 
toms in youth with $\mathrm{OV} / \mathrm{OB}$, and the possibility that these symptoms will lead to further weight gain and psychological problems, it is imperative to measure symptoms of disordered eating in research and practice. However, such measurement is challenging due to the possibility that youth might misunderstand questions with technical or abstract language or fail to make distinctions that researchers and clinicians are interested in assessing (e.g., between deliberate and illness-induced vomiting).

Structured clinical interviews can address many of these challenges and yield high quality data about youths' disordered eating symptoms (Garfinkel and Newman, 2001; Tanofsky-Kraff et al., 2003), but unfortunately can be timeconsuming and expensive and typically require specialized training to administer and interpret. As a result, researchers and clinicians often use self-report questionnaires to expediently measure disordered eating symptoms, either as part of a two-stage process (i.e., by interviewing only those who score highly on self-report measures) or as their sole assessment (Tanofsky-Kraff et al., 2003).

The most widely used standardized measure of disordered eating symptoms is the Eating Attitudes Test (EAT), which was developed in the 1970 s to measure the symptoms and concerns characteristic of anorexia nervosa and bulimia nervosa in adults (Garner and Garfinkel, 1979). The 26-item short-form of the EAT (Garner et al., 1982) has been adapted for use with children and adolescents by simplifying its language; this modified form is called the Children's Eating Attitudes Test (ChEAT) (Maloney et al., 1988). This measure has seen widespread use and has been translated into numerous international languages including (but not limited to) Croatian (Ambrosi-Randic and Pokrajac-Bulian, 2005), Dutch (Theuwis et al., 2009), Japanese (Mukai and McCloskey, 1996), and Spanish (Rojo-Moreno et al., 2011).

The ChEAT has also seen widespread use as a measure of disordered eating symptoms among youth with $\mathrm{OV} / \mathrm{OB}$ (Croker et al., 2012; Edwards et al., 2006; FollansbeeJunger et al., 2010; Gowey et al., 2014, 2017; Marcus et al., 2009; Ranzenhofer et al., 2008; Saelens et al., 2002; Tanofsky-Kraff et al., 2006, 2003; Theuwis et al., 2009; Tyler and Horner, 2016; Vander Wal, 2004; Wildes et al., 2010; Willmer et al., 2016). Nearly all of this work cites the following two studies as evidence of the ChEAT's reliability and validity. Maloney et al. (1988) examined 318 third through sixth graders (53\% female, $92 \%$ White) and found an overall coefficient alpha of 0.76 , as well as a three-week test-retest correlation of 0.81 for a subset of 68 children. Smolak and Levine (1994) examined 308 sixth through eighth graders (100\% female, $100 \%$ White) and found an overall coefficient alpha of 0.87 and positive correlations with concurrent measures of weight management behavior $(r=.36)$ and body dissatisfaction $(r=.39)$. Additionally, studies on $\mathrm{OV} / \mathrm{OB}$ have reported high values of coefficient alpha in their own samples to support its reliability (Gowey et al., 2014, 2017; Ranzenhofer et al., 2008; Saelens et al., 2002; Theuwis et al., 2009; Tyler and Horner, 2016; Vander Wal, 2004).

However, there are several issues with interpreting these (and similar) findings in this way. First, the psychometric properties of scales can vary between populations (Meredith, 1993; Vandenberg and Lance, 2000), and therefore evidence of reliability and validity in unselected samples of school children (e.g., Maloney et al., 1988; Smolak and Levine, 1994) may not generalize to samples of youth with OV/OB (who are also likely to be more diverse). Second, a long history of psychometric research has demonstrated that coefficient alpha is not a measure of internal consistency or structure and is only an estimate of reliability in conditions that are unlikely to occur in practice (Cortina, 1993; Green et al., 1977; Hattie, 1985; Schmitt, 1996; Sijtsma, 2009). Finally, a critical assumption of these reliability and validity analyses remains untested: that the ChEAT total score is essentially unidimensional (i.e., measuring a single underlying construct). As Hattie (1985) stated, "to make psychological sense when relating variables, ordering persons on some attribute, forming groups on the basis of some variable, or making comments about individual differences, the variable must be unidimensional" (p. 139). Thus, despite widespread use in samples with OV/OB, it is not clear that the ChEAT has adequate psychometric properties in this population.

The current study addresses this gap in the literature by conducting a modern psychometric analysis of the ChEAT as applied to youth with OV/OB. This analysis is based on bifactor modeling, which can assess the unidimensionality of a measure as well as many other psychometric properties (Reise et al., 2013; Rodriguez et al., 2016b). A bifactor model assumes that the correlations among items can be accounted for by a general factor representing shared variance among all items and a set of group factors where variance over and above the general factor is shared among subsets of items presumed to be highly similar in content. The general factor represents the broad central construct an instrument intends to measure (e.g., disordered eating symptoms), whereas group factors represent more conceptually specific subdomain constructs (e.g., dieting, purging, and body dissatisfaction). By examining the general and group factors, and the proportion of variance account for by each, many practical psychometric questions can be answered.

Is the ChEAT measuring one underlying construct? How much of the variance in total and subscale scores is reliable? How well does the total score measure a single underlying construct? How unique is the variance being captured by subscale scores? Would "optimally weighted" scores (e.g., factor score estimates or structural equation modeling) perform better than unit-weighted scores? The goal of the current study is to answer to these questions and to provide recommendations about the ChEAT in youth with OV/OB. 
Table 1

\begin{tabular}{|c|c|c|c|}
\hline Variable & Combined & Sample 1 & Sample 2 \\
\hline Number & 400 & 131 & 269 \\
\hline Age in years* & $11.24(2.25)$ & $13.05(2.58)$ & $10.36(1.39)$ \\
\hline \multicolumn{4}{|l|}{ Gender } \\
\hline Female $^{\dagger}$ & $214(54 \%)$ & $69(53 \%)$ & $145(54 \%)$ \\
\hline Male $^{\dagger}$ & $185(46 \%)$ & $62(47 \%)$ & $123(46 \%)$ \\
\hline \multicolumn{4}{|l|}{ Race } \\
\hline Black $^{\dagger}$ & $115(29 \%)$ & $80(61 \%)$ & $35(13 \%)$ \\
\hline White $^{\dagger}$ & $229(57 \%)$ & $45(34 \%)$ & $184(68 \%)$ \\
\hline Other $^{\dagger}$ & $56(14 \%)$ & $6(5 \%)$ & $50(19 \%)$ \\
\hline \multicolumn{4}{|l|}{ Weight Status } \\
\hline Overweight ${ }^{\dagger}$ & $27(7 \%)$ & $4(3 \%)$ & $23(9 \%)$ \\
\hline Obesity $^{\dagger}$ & $373(93 \%)$ & $127(97 \%)$ & $246(91 \%)$ \\
\hline BMI z-score* & $2.31(0.41)$ & $2.56(0.36)$ & $2.19(0.37)$ \\
\hline
\end{tabular}

* Mean (Standard Deviation) $\quad$ Count (Percentage)

\section{Methods}

\section{Sample 1}

Youth with OV/OB were recruited from a multidisciplinary pediatric obesity clinic in the southern United States (Anderson et al., 2018). Data collection in the clinic is ongoing, so we examined all youth enrolled prior to April 13, 2018. This yielded a sample of 131 youth between the ages of 8 and 18, all of whom were above the 85th percentile on BMI for age and sex (Kuczmarski et al., 2000). Table 1 provides additional information about this sample. Patients in the clinic were referred for a psychological evaluation by medical providers if emotional or behavioral disorders and/or disordered eating symptoms were suspected. Evaluations consisted of a clinical interview with the clinic psychologist and the completion of self-report questionnaires. Caregivers and youth provided consent and assent, respectively, for the evaluation. The examination of clinical data for research purposes was approved by the governing institutional review board.

\section{Sample 2}

Youth with OV/OB were recruited from rural communities in the southern United States to participate in a randomized controlled weight-management trial (Janicke et al., 2011). Families interested in participating contacted the research team and completed telephone and in-person screenings to confirm eligibility. Data examined in this study were completed by youth during baseline assessments that were conducted prior to the start of weight-management treatment. The sample consisted of 269 youth between the ages of 8 and 12 , all of whom were above the 85 th percentile on BMI based on age and sex. Table 1 provides additional information about this sample. The larger randomized controlled trial was approved by the governing institutional review board.

\section{Measures}

The Children's Eating Attitudes Test (ChEAT) is a 26item self-report questionnaire designed to assess disordered eating attitudes and behaviors in youth (Maloney et al., 1988). Items are declarative statements about eating, which are rated on a six-point ordered scale using the following response categories: never, rarely, sometimes, often, very often, and always. Based on the scoring procedure of the EAT (Garner and Garfinkel, 1979), the three least-symptomatic response categories are collapsed and assigned a value of 0 , whereas the most-symptomatic response category is assigned a value of 3 , the second most-symptomatic is assigned a value of 2, and the third most-symptomatic is assigned a value of 1 . All items are phrased such that always is the most-symptomatic response category except for item \#25 ("I enjoy trying new, rich foods"), which is reverse-scored.

\section{Data Analysis Plan}

All analyses were completed in the R statistical computing environment (R Core Team, 2018) using the psych package (Revelle, 2018). First, basic descriptive analyses were used to examine the missing data patterns and distributional properties of the individual ChEAT item variables. To account for missing data, the item correlation matrix was estimated using the corFiml function, which uses full information maximum likelihood (Enders, 2010). Then several bifactor models, with between three and seven group factors, were estimated from that correlation matrix using the omegaSem function. This function first estimates an exploratory bifactor model and then estimates a confirmatory bifactor model using the highest loading items on each factor. These models were compared on several model fit indexes ( $\mathrm{Hu}$ and Bentler, 1999): the Comparative Fit Index (CFI), TuckerLewis Index (TLI), Root Mean Squared Error of Approximation (RMSEA), and Standardized Root Mean Residual (SRMR). Psychometric coefficients were derived from the best-fitting model and used to answer practical questions.

\section{Psychometric Coefficients}

Numerous psychometric coefficients can be derived from a bifactor model. Most are based on the idea of partitioning the observed variance in unit-weighted scores according to the following sources: general factor, group factor, and residual (i.e., item-specific and error) variance. This logic can be applied to the observed variance in either all items or only the items comprising a single subscale.

Is the ChEAT measuring one underlying construct or many? This question can be answered by the explained common variance (ECV) coefficient, which estimates the propor- 
tion of the modeled common (i.e., general and group factor) variance that is attributable to the general factor, and the percentage of uncontaminated correlations (PUC) coefficient, which is the number of correlations between items from different group factors divided by the total number of correlations between items. When ECV and PUC are both high $(>.70)$, the common variance can be regarded as essentially unidimensional (Reise, 2012; Rodriguez et al., 2016a).

How much of the variance in total and subscale scores is reliable? This question can be answered by the omega total coefficient, which estimates the proportion of variance in unit-weighted total (or subscale) scores that is attributable to the general factor and all group factors. High omega total values indicate that the total (or subscale) scores contain little residual variance. When applied to all items, omega total is called the omega coefficient $(\omega)$, and when applied to the items comprising a single subscale, omega total is called omega subscale $\left(\omega_{S}\right)$.

How well does the total score measure a single broad, central construct? How much are subscale scores confounded by the general factor? These questions can be answered by the omega general coefficient, which estimates the proportion of variance in unit-weighted total (or subscale) scores that is attributable to the general factor only. High omega general values indicate that the general factor is the dominant source of systematic variance. When applied to all items, omega general is called omega hierarchical $\left(\omega_{H}\right)$. High $\omega_{H}$ values $(>.80)$ suggest that the unit-weighted total score can be interpreted as a "univocal" indicator of a single latent variable (Rodriguez et al., 2016a). When applied to the items comprising a single subscale, omega general is an indication of how much the subscale scores are influenced/confounded by the general factor.

How much is the total score confounded by construct multidimensionality? How well do the subscale scores measure their specific subdomain construct? These questions can be answered by the omega group coefficient, which estimates the proportion of variance in unit-weighted total (or subscale) scores that is attributable to the group factor(s) only. High omega group values indicate that the group factor(s) are the dominant source of systematic variance. When applied to all items, omega group indicates how much the unit-weighted total score is influenced/confounded by group factor variance (i.e., multidimensionality) as opposed to the general factor. When applied to the items comprising a single subscale, omega group is called omega hierarchical subscale $\left(\omega_{H S}\right)$. When $\omega_{H S}$ is high relative to the corresponding $\omega_{S}$, much of the reliable variance in unit-weighted subscale scores is unique to that group factor.

The omega-related coefficients provide information about the psychometric properties and utility of unit-weighted composite (i.e., sum) scores, which is how the ChEAT is typically used. However, it is also possible to estimate "op-
Table 2

Model comparison using multiple fit measures

\begin{tabular}{lcccc}
\hline Model & CFI $^{*}$ & TLI $^{*}$ & RMSEA $^{\dagger}$ & SRMR $^{\dagger}$ \\
\hline 3 Group Factors & .796 & .757 & .061 & .061 \\
4 Group Factors & .833 & .802 & .055 & .070 \\
5 Group Factors & .846 & .816 & .053 & .063 \\
6 Group Factors & .841 & .811 & .054 & .064 \\
7 Group Factors & .830 & .797 & .055 & .070 \\
\hline
\end{tabular}

${ }^{*}$ Incremental fit index (higher is better)

${ }^{\dagger}$ Absolute fit index (lower is better)

timally weighted" factor scores or to develop a measurement model using structural equation modeling (Bollen, 1989).

How would optimally weighted factor score estimates or structural equation modeling perform, as opposed to unit-weighted total or subscale scores? The factor determinacy (FD) coefficient evaluates the degree to which researchers can confidently assume that individual differences on factor score estimates are good representations of true individual differences on the factor. It has been recommended (Gorsuch, 1983) that factor score estimates only be considered when FD is high $(>.90)$. The construct replicability (H) coefficient evaluates how well a set of items represents a latent variable and how likely the measurement model is to replicate across studies. High $\mathrm{H}$ values $(>.80)$ indicate that a latent-variable is well-defined and likely to be stable across studies (Rodriguez et al., 2016a). Both coefficients can be applied to the general factor as well as to group factors.

\section{Results}

In Sample 1, missing data were rare; most participants $(67 \%)$ had complete data on all items, and the mean number of missing items per participant was $0.66(S D=1.49)$. In Sample 2, missing data patterns were not analyzable because the raw data, prior to mean imputation, was no longer available. In the combined sample, the average item distribution was positively skewed ( $M=2.76, S D=2.13$ ) after implementing the four-category scoring procedure.

To determine the most appropriate measurement model for the ChEAT, we estimated and compared confirmatory bifactor models with between three and seven group factors. Table 2 provides two incremental fit indexes and two absolute fit indexes for each model (Hu and Bentler, 1999). According to all four fit indexes, the model with five group factors had the best fit and was further analyzed.

Table 3 presents each item's factor loadings on the general factor and group factors from the selected model. It shows that 17 items loaded on both the general factor and a group factor, 3 items loaded on the general factor only, 4 items loaded on a group factor only, and 2 items did not load on the general factor or any group factors. The first group factor 
Table 3

Factor loadings on the general factor $(G)$ and group factors (F1-F5)

\begin{tabular}{|c|c|c|c|c|c|c|c|}
\hline Item & Text Summary & G & $\mathrm{F} 1$ & $\mathrm{~F} 2$ & F3 & $\mathrm{F} 4$ & F5 \\
\hline \#01 & I am scared about being overweight & .53 & - & - & .20 & - & - \\
\hline$\# 02$ & I stay away from eating when I am hungry & .23 & .26 & - & - & - & - \\
\hline \#03 & I think about food a lot of the time & .28 & - & .69 & - & - & - \\
\hline \#04 & I have gone on eating binges... & .42 & - & .39 & - & - & - \\
\hline \#05 & I cut my food into small pieces & - & .22 & - & - & - & - \\
\hline \#06 & I am aware of the calorie content in foods... & .23 & .43 & - & - & - & - \\
\hline$\# 07$ & I try to stay away from foods such as breads... & .28 & .52 & - & - & - & - \\
\hline \#08 & I feel that others would like me to eat more & .20 & - & - & - & - & .50 \\
\hline \#09 & I vomit after I have eaten & .35 & - & - & - & .54 & - \\
\hline$\# 10$ & I feel very guilty after eating & .60 & - & - & - & - & - \\
\hline$\# 11$ & I think a lot about wanting to be thinner & .45 & - & - & .84 & - & - \\
\hline$\# 12$ & I think about burning up calories when I exercise... & .38 & - & - & .31 & - & - \\
\hline \#13 & Other people think I'm too thin & - & - & - & - & - & .48 \\
\hline$\# 14$ & I think a lot about having fat on my body & .45 & - & - & .32 & - & - \\
\hline$\# 15$ & I take longer than others to eat my meals & .28 & - & - & - & - & - \\
\hline \#16 & I stay away from foods with sugar in them & - & .62 & - & - & - & - \\
\hline$\# 17$ & I eat diet foods & .25 & .72 & - & - & - & - \\
\hline \#18 & I think that food controls my life & .42 & - & .34 & - & - & 一 \\
\hline \#19 & I can show self control around food & - & .32 & - & - & - & - \\
\hline$\# 20$ & I feel that others pressure me to eat & .21 & - & - & - & - & .37 \\
\hline \#21 & I give too much time and thought to food & .50 & - & .46 & - & - & - \\
\hline \#22 & I feel uncomfortable after eating sweets & .48 & - & - & - & - & - \\
\hline \#23 & I have been dieting & .23 & .73 & - & - & - & - \\
\hline \#24 & I like my stomach to be empty & - & - & - & - & - & - \\
\hline \#25 & I enjoy trying new, rich foods (reverse-coded) & - & - & - & - & - & - \\
\hline \#26 & I have the urge to vomit after eating & .43 & - & - & - & 63 & - \\
\hline
\end{tabular}

Table 4

Psychometric properties of the general and group factors

\begin{tabular}{lllllll}
\hline & $\mathrm{G}$ & $\mathrm{F} 1$ & $\mathrm{~F} 2$ & $\mathrm{~F} 3$ & $\mathrm{~F} 4$ & $\mathrm{~F} 5$ \\
\hline ECV & .36 & - & - & - & - & - \\
PUC & .90 & - & - & - & - & - \\
$\omega_{\text {total }}$ & .83 & .74 & .72 & .66 & .67 & .47 \\
$\omega_{\text {general }}$ & .58 & .10 & .31 & .53 & .21 & .09 \\
$\omega_{\text {group }}$ & .24 & .64 & .41 & .13 & .46 & .37 \\
FD & .86 & .98 & .82 & .94 & .78 & .71 \\
H & .70 & .80 & .62 & .57 & .61 & .50 \\
\hline
\end{tabular}

(F1) seemed to capture dieting, the second group factor (F2) seemed to capture food preoccupation, the third group factor (F3) seemed to capture body weight concerns, the fourth group factor (F4) seemed to capture purging, and the fifth group factor (F5) seemed to capture social pressure to eat. While not identical, these group factors are similar to previously proposed ChEAT factors (Anton et al., 2006; Ranzenhofer et al., 2008).

Numerous psychometric coefficients were derived from the selected model and are presented in Table 4 and Figure 1. The ECV and PUC coefficients together did not support a unidimensional interpretation of the common variance.

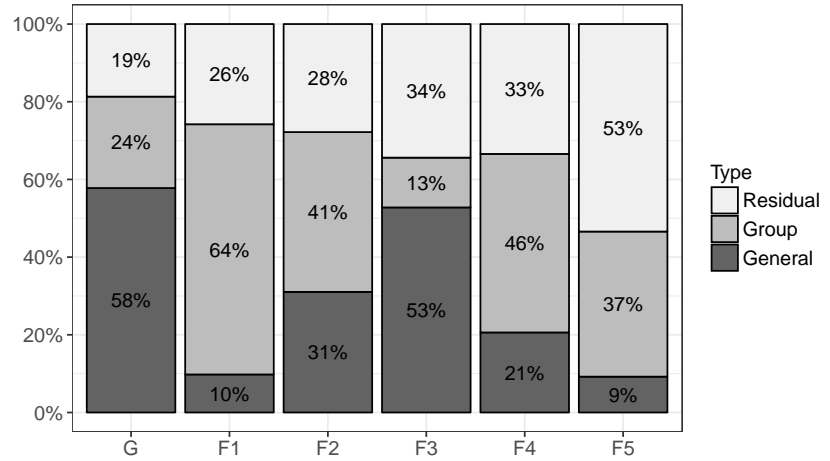

Figure 1. Partitioning of variance in total and subscale scores

Comparison of the omega-related coefficients for the general factor revealed that the unit-weighted total score contained $83 \%$ reliable variance $(\omega)$ and $17 \%$ residual variance; the reliable variance can be further partitioned into $58 \%$ general factor variance $\left(\omega_{H}\right)$ and $24 \%$ group factor variance. The FD coefficient did not support the use of factor score estimates for the general factor, and the $\mathrm{H}$ coefficient did not support the use of structural equation modeling for the general factor.

Comparison of the omega-related coefficients for the group factors reveals that the unit-weighted subscale scores 
contained varying amounts of reliable variance $\left(\omega_{S}\right)$. The first and second group factors contained around $73 \%$ reliable variance, the third and fourth group factors contained around $66 \%$ reliable variance, and the fifth group factor contained $47 \%$ reliable variance. This reliable variance can be further partitioned into varying amounts of group factor variance $\left(\omega_{H S}\right)$ and general factor variance. The first group factor (dieting) contained mostly group factor variance, which supports its use as a subscale, but the remaining group factors contained a mixture of group factor and general factor variance. The subscale FD coefficients supported the use of factor score estimates for the first group factor (dieting) and the third group factor (body weight concerns). The subscale $\mathrm{H}$ coefficients supported the use of measurement models for the first group factor only (dieting).

\section{Discussion}

Pediatric OV/OB is a serious global health concern affecting a large, and growing, proportion of youth. Research suggests that these conditions have bidirectional relationships with mental health issues including disordered eating symptoms (e.g., body dissatisfaction, binge eating, and unhealthy weight-control strategies) (Goldschmidt et al., 2008). However, the self-report measure most commonly used to measure disordered eating symptoms in youth - the Children's Eating Attitudes Test (ChEAT) - has not been psychometrically analyzed in samples consisting of only youth with OV/OB. The current study performed such an analysis using a large and diverse sample of 400 treatment-seeking youth with OV/OB from the southern United States.

Our results do not support the use of the ChEAT as a unidimensional scale in this population. First, rather than measuring a single underlying construct with several nuanced but largely overlapping sub-constructs, in this population, the ChEAT appears to be measuring multiple constructs with inadequate overlap $(\mathrm{ECV}<.70)$. Second, the unit-weighted total score (in which scores on each item are added together) cannot be considered a reasonable approximation of the overlap that does exist $\left(\omega_{H}<.80\right)$. Finally, using the "optimally weighted" total score (e.g., factor score estimates or structural equation modeling) would not fully alleviate these problems, as these scores are not likely to replicate across studies $(\mathrm{FD}<.90$ and $\mathrm{H}<.80)$. It is important to note that these results were found in the context of confirmatory bifactor modeling, which forces cross-loaded variance into the general factor and therefore provides an especially optimistic estimate of the overlap between subdomain constructs.

The results regarding ChEAT subscales were mixed. Although five potential subscales were identified, only one contained a majority of unique (i.e., group factor) variance and would be replicable using factor score estimates or structural equation modeling $(\mathrm{FD}>.90, \mathrm{H}>.80)$. This subscale contained eight items about dieting.
These findings raise a number of important theoretical questions as we try to account for the ChEAT's inadequate performance in this sample. Are these results an indictment of the scale itself? Perhaps a different measure, derived from different items and different methods (e.g., item response theory), would fare better at generalizing across groups. Or are these results suggestive of a deeper issue with the construct of disordered eating symptoms? Perhaps no unidimensional measure of disordered eating attitudes and behaviors is even possible because the construct itself is not unitary. Perhaps dieting, purging, food preoccupation, body weight concern, and eating concerns (or some subset of these concepts) are different things that vary independently of one another. Finally, are these issues with the ChEAT confined to individuals with OV/OB or do they extend to other populations too? Perhaps the reliance on coefficient alpha in previous research has masked psychometric issues even in the populations that the ChEAT was initially developed for. More research is needed to explore these possibilities. In particular, it would be revealing to apply bifactor modeling to the ChEAT in samples with normal weight and underweight, as well as to compare it to alternative measures.

These findings have a number of practical implications as well. First, we recommend that researchers and clinicians working with pediatric populations with OV/OB look for more psychometrically sound alternatives to the ChEAT. In particular, validated measures of subcomponents of the disordered eating construct (e.g., body dissatisfaction, unhealthy dieting, and binge eating) are recommended until the aforementioned theoretical questions can be answered. Second, we recommend that researchers move beyond coefficient alpha as a measure of reliability. Luckily, the alternatives presented in this study are easy to calculate using freely available software (Revelle, 2018). Finally, we recommend that researchers developing novel instruments pay close attention to the principles of test construction (Ziegler, 2014) and validation (Kane, 2013), as well as consider making instruments open source and freely available (Tackett et al., 2017), as the ChEAT's availability was likely one of reasons it has become so widely used.

Our results should be interpreted considering several study limitations. First, the combined sample was largely composed of youth between 9 and 14 years old with obesity. Therefore, our results may not generalize to older youth or those with overweight. Second, although our sample was quite diverse in terms of race, both samples were from the southern United States and used the English-language version of the ChEAT. Therefore, our results may not generalize as well to youth living in other areas or completing other versions of the scale. Finally, it was not ideal that missing data in Sample 2 was addressed using mean imputation. Fortunately, this approach is unlikely to have affected our conclusions because its most likely consequence on bifactor 
modeling would have been to inflate the general factor and therefore to overestimate the unidimensionality and omega general coefficients.

In summary, the ChEAT displayed marked psychometric problems when applied in a large and diverse sample of treatment-seeking youth with $\mathrm{OV} / \mathrm{OB}$. Its common variance was not unidimensional, its unit-weighted total score was not a reliable indicator of a broad central construct, and its optimally weighted total score was not likely to replicate across studies. Several subscales (i.e., dieting and purging) fared somewhat better but would require optimally weighted subscale scores and, even then, barely met acceptability criteria. We recommend using alternatives to the ChEAT when conducting research and practice with youth with $\mathrm{OV} / \mathrm{OB}$, and researchers are encouraged to revisit the construct of disordered eating symptoms to assess the extent to which the empirical and theoretical evidence supports this construct's unidimensionality in all populations (e.g., across ages, weight groups, and disorders).

\section{Acknowledgements}

CL's work has been funded by the Department of Psychiatry and Human Behavior and the Department of Pediatrics at the University of Mississippi Medical Center. MG's work has been funded by the University of Alabama at Birmingham and grant \#K12HS023009 from the Agency for Healthcare Research and Quality. DJ's work has been funded by grant \#R18DK082374 from the National Institute of Diabetes and Digestive and Kidney Diseases.

\section{Author Contributions}

CL collected sample 1. DJ, MG, and CL collected sample 2. JG and CL conducted the literature search, conceived the study, and wrote the manuscript. JG conceived the experiments, analyzed and interpreted the data, and generated the figures and tables. All authors were involved in editing the manuscript and had final approval of the submitted and published versions.

\section{Supplemental Materials}

All analyses (i.e., input syntax as R markdown files and formatted output as HTML notebook files), as well as the estimated correlation matrix (from which all analyses can be recreated) are available on the Open Science Framework: https://osf.io/5y2ke/

\section{References}

Ambrosi-Randic, N. and Pokrajac-Bulian, A. (2005). Psychometric properties of the Eating Attitudes Test and Children's Eating Attitudes Test in Croatia. Eating and Weight Disorders, 10(4):e76-82.
Anderson, L. M., Wong, N., Lanciers, S., and Lim, C. S. (2018). The relative importance of social anxiety facets on disordered eating in pediatric obesity. Eating and Weight Disorders - Studies on Anorexia, Bulimia and Obesity.

Anton, S. D., Han, H., Newton, R. L., Martin, C. K., York-Crowe, E., Stewart, T. M., and Williamson, D. A. (2006). Reformulation of the Children's Eating Attitudes Test (ChEAT): Factor structure and scoring method in a non-clinical population. Eating and Weight Disorders, 11(4):201-210.

Bollen, K. A. (1989). Structural equations with latent variables. Wiley, New York, NY.

Cortina, J. M. (1993). What is coefficient alpha? An examination of theory and applications. Journal of Applied Psychology, 78(1):98-104.

Croker, H., Viner, R. M., Nicholls, D., Haroun, D., Edwards, C., and Wells, J. C. K. (2012). Family-based behavioural treatment of childhood obesity in a UK National Health Service setting: Randomised controlled trial. International Journal of Pediatric Obesity, 36(1):1-19.

Daniels, S. R. (2006). The consequences of childhood overweight and obesity. The Future of Children, 16(1):47-67.

Edwards, C., Nicholls, D., Croker, H., Van Zyl, S., Viner, R., and Wardle, J. (2006). Family-based behavioural treatment of obesity: Acceptability and effectiveness in the UK. European Journal of Clinical Nutrition, 60(5):587592.

Enders, C. K. (2010). Applied missing data analysis. Guilford Press, New York, NY.

Follansbee-Junger, K., Janicke, D. M., and Sallinen, B. J. (2010). The influence of a behavioral weight management program on disordered eating attitudes and behaviors in children with overweight. Journal of the American Dietetic Association, 110(11):1653-1659.

Garfinkel, P. E. and Newman, A. (2001). The eating attitudes test: Twenty-five years later. Eating and Weight Disorders, 6(1):1-21.

Garner, D. M. and Garfinkel, P. E. (1979). The eating attitudes test: An index of the symptoms of anorexia nervosa. Psychological Medicine, 9:273-279.

Garner, D. M., Olmsted, M. P., Bohr, Y., and Garfinkel, P. E. (1982). The eating attitudes test: Psychometric features and clinical correlates. Psychological Medicine, 12:871878. 
Goldschmidt, A. B., Aspen, V. P., Sinton, M. M., TanofskyKraff, M., and Wilfley, D. E. (2008). Disordered eating attitudes and behaviors in overweight youth. Obesity, 16(2):257-264.

Gorsuch, R. L. (1983). Factor analysis. Erlbaum, Hillsdale, NJ.

Gowey, M. A., Lim, C. S., Clifford, L. M., and Janicke, D. M. (2014). Disordered eating and health-related quality of life in overweight and obese children. Journal of Pediatric Psychology, 39(5):552-561.

Gowey, M. A., Stromberg, S. E., Lim, C. S., and Janicke, D. M. (2017). The moderating role of body dissatisfaction in the relationship between ADHD symptoms and disordered eating in pediatric overweight and obesity. Children's Health Care, 46(1):15-33.

Green, S. B., Lissitz, R. W., and Mulaik, S. A. (1977). Limitations of coefficient alpha as an index of unidimesionality. Educational and Psychological Measurement, 37:827-838.

Hattie, J. (1985). Methodology review: Assessing unidimensionality of tests and items. Applied Psychological Measurement, 9(2):139-164.

Heinberg, L. J., Thompson, J. K., and Matzon, J. L. (2001). Body image dissatisfaction as a motivator for healthy lifestyle change: Is some distress beneficial? In StriegelMoore, R. H. and Smolak, L., editors, Eating disorders: Innovative directions in research and practice, pages 215 232. American Psychological Association.

Hu, L. and Bentler, P. M. (1999). Cutoff criteria for fit indexes in covariance structure analysis: Conventional criteria versus new alternatives. Structural Equation Modeling, 6:1-55.

Janicke, D. M., Lim, C. S., Perri, M. G., Bobroff, L. B., Mathews, A. E., Brumback, B. A., Dumont-Driscoll, M., and Silverstein, J. H. (2011). The extension family lifestyle intervention project (E-FLIP for Kids): Design and methods. Contemporary Clinical Trials, 32(1):50-58.

Kane, M. T. (2013). Validating the interpretations and uses of test scores. Journal of Educational Measurement, 50(1):1-73.

Kotler, L. A., Cohen, P., Davies, M., Pine, D. S., and Walsh, B. T. (2001). Longitudinal relationships between childhood, adolescent, and adult eating disorders. Journal of the American Academy of Child and Adolescent Psychiatry, 40(12):1434-1440.
Kuczmarski, R. J., Ogden, C. L., Grummer-strawn, L. M., Flegal, K. M., Guo, S. S., Wei, R., Mei, Z., Curtin, L. R., Roche, A. F., and Johnson, C. L. (2000). CDC growth charts: United States. Advance Data, 314:1-27.

Maloney, M. J., McGuire, J. B., and Daniels, S. R. (1988). Reliability testing of a children's version of the eating attitude test. Journal of the American Academy of Child $\mathcal{E}$ Adolescent Psychiatry, 27(5):541-543.

Marcus, C., Nyberg, G., Nordenfelt, A., Karpmyr, M., Kowalski, J., and Ekelund, U. (2009). A 4-year, cluster-randomized, controlled childhood obesity prevention study: STOPP. International Journal of Obesity, 33(4):408-417.

Meredith, W. (1993). Measurement invariance, factor analysis and factorial invariance. Psychometrika, 58(4):525543.

Mukai, T. and McCloskey, L. A. (1996). Eating attitudes among Japanese and American elementary schoolgirls. Journal of Cross-Cultural Psychology, 27(4):424-435.

Neumark-Sztainer, D., Hannan, P. J., Story, M., and Perry, C. L. (2004). Weight-control behaviors among adolescent girls and boys: Implications for dietary intake. Journal of the American Dietetic Association, 104(6):913-920.

Neumark-Sztainer, D., Story, M., Hannan, P. J., Perry, C. L., and Irving, L. M. (2002). Weight-related concerns and behaviors among overweight and nonoverweight adolescents: Implications for preventing weight-related disorders. Archives of Pediatrics $\mathcal{E}$ Adolescent Medicine, 156(2):171-178.

Neumark-Sztainer, D., Wall, M., Guo, J., Story, M., Haines, J., and Eisenberg, M. (2006). Obesity, disordered eating, and eating disorders in a longitudinal study of adolescents: How do dieters fare 5 years later? Journal of the American Dietetic Association, 106(4):559-568.

R Core Team (2018). R: A language and environment for statistical computing. R Foundation for Statistical Computing, Vienna, Austria.

Ranzenhofer, L. M., Tanofsky-Kraff, M., Menzie, C. M., Gustafson, J. K., Rutledge, M. S., Keil, M. F., Yanovski, S. Z., and Yanovski, J. A. (2008). Structure analysis of the Children's Eating Attitudes Test in overweight and at-risk for overweight children and adolescents. Eating Behaviors, 9(2):218-227.

Reise, S. P. (2012). The rediscovery of bifactor measurement models. Multivariate Behavioral Research, 47(5):667696. 
Reise, S. P., Bonifay, W. E., and Haviland, M. G. (2013). Scoring and modeling psychological measures in the presence of multidimensionality. Journal of Personality Assessment, 95(2):129-140.

Revelle, W. (2018). psych: Procedures for psychological, psychometric, and personality research. Northwestern University, Evanston, Illinois.

Rodriguez, A., Reise, S. P., and Haviland, M. G. (2016a). Applying bifactor statistical indices in the evaluation of psychological measures. Journal of Personality Assessment, 98(3):223-237.

Rodriguez, A., Reise, S. P., and Haviland, M. G. (2016b). Evaluating bifactor models: Calculating and interpreting statistical indices. Psychological Methods, 21(2):137150.

Rojo-Moreno, L., Garcia-Miralles, I., Plumed, J., Barbera, M., Morales, M. M., Ruiz, E., and Livianos, L. (2011). Children's eating attitudes test: Validation in a sample of Spanish schoolchildren. International Journal of Eating Disorders, 44(6):540-546.

Saelens, B. E., Sallis, J. F., Wilfley, D. E., Patrick, K., Cella, J. A., and Buchta, R. (2002). Behavioral weight control for overweight adolescents initiated in primary care. Obesity Research, 10(1):22-32.

Sahoo, K., Sahoo, B., Choudhury, A., Sufi, N., Kumar, R., and Bhadoria, A. (2015). Childhood obesity: Causes and consequences. Journal of Family Medicine and Primary Care, 4(2): 187.

Schmitt, N. (1996). Uses and abuses of coefficient alpha. Psychological Assessment, 8(4):350-353.

Sijtsma, K. (2009). On the use, the misuse, and the very limited usefulness of Cronbach's alpha. Psychometrika, 74(1):107-120.

Skinner, A. C., Ravanbakht, S. N., Skelton, J. A., Perrin, E. M., and Armstrong, S. C. (2018). Prevalence of obesity and severe obesity in US children, 1999-2016. Pediatrics, 141(3):e20173459.

Smolak, L. and Levine, M. P. (1994). Psychometric properties of the children's eating attitudes test. International Journal of Eating Disorders, 16(3):275-282.

Tackett, J. L., Lilienfeld, S. O., Patrick, C. J., Johnson, S. L., Krueger, R. F., Miller, J. D., Oltmanns, T. F., and Shrout, P. E. (2017). It's time to broaden the replicability conversation: Thoughts for and from clinical psychological science. Perspectives on Psychological Science, 12(5):742756.
Tanofsky-Kraff, M., Cohen, M. L., Yanovski, S. Z., Cox, C., Theim, K. R., Keil, M., Reynolds, J. C., and Yanovski, J. A. (2006). A prospective study of psychological predictors of body fat gain among children at high risk for adult obesity. Pediatrics, 117(4):1203-1209.

Tanofsky-Kraff, M., Morgan, C. M., Yanovski, S. Z., Marmarosh, C., Wilfley, D. E., and Yanovski, J. A. (2003). Comparison of assessments of children's eatingdisordered behaviors by interview and questionnaire. International Journal of Eating Disorders, 33(2):213-224.

Theuwis, L., Moens, E., and Braet, C. (2009). Psychometric quality of the Dutch version of the Children's Eating Attitude Test in a community sample and a sample of overweight youngsters. Psychologica Belgica, 49(4):311-330.

Tyler, D. O. and Horner, S. D. (2016). A primary care intervention to improve weight in obese children: A feasibility study. Journal of the American Association of Nurse Practitioners, 28(2):98-106.

Vandenberg, R. J. and Lance, C. E. (2000). A review and synthesis of the measurement invariance literature: Suggestions, practices, and recommendations for organizational research. Organizational Research Methods, 3(1):4-70.

Vander Wal, J. S. (2004). Eating and body image concerns among average-weight and obese African American and Hispanic girls. Eating Behaviors, 5(2):181-187.

Wang, Y. and Lobstein, T. (2006). Worldwide trends in childhood overweight and obesity. International Journal of Pediatric Obesity, 1(1):11-25.

Wildes, J. E., Marcus, M. D., Kalarchian, M. A., Levine, M. D., Houck, P. R., and Cheng, Y. (2010). Self-reported binge eating in severe pediatric obesity: Impact on weight change in a randomized controlled trial of family-based treatment. International Journal of Obesity, 34(7):11431148 .

Willmer, M., Berglind, D., Tynelius, P., Ghaderi, A., Näslund, E., and Rasmussen, F. (2016). Changes in eating behaviour and food choices in families where the mother undergoes gastric bypass surgery for obesity. European Journal of Clinical Nutrition, 70(1):35-40.

Zabinski, M. F., Saelens, B. E., Stein, R. I., Hayden-Wade, H. A., and Wilfley, D. E. (2003). Overweight children's barriers to and support for physical activity. Obesity Research, 11(2):238-246.

Ziegler, M. (2014). Stop and state your intentions!: Let's not forget the $\mathrm{ABC}$ of test construction. European Journal of Psychological Assessment, 30(4):239-242. 